\title{
Strange Objects in the Complex Plane
}

\author{
Michael Widom, ${ }^{1}$ David Bensimon, ${ }^{1}$ Leo P. Kadanoff, ${ }^{1}$ \\ and Scott J. Shenker ${ }^{1}$
}

Received April 18, 1983

\begin{abstract}
Julia sets are examined as examples of strange objects which arise in the study of long time properties of simple dynamical systems. Technically they are the closure of the set of unstable cycles of analytic maps. Physically, they are sets of points which lead to chaotic behavior. The map $f(z)=z^{2}+p$ is analyzed for small $p$ where the Julia set is a closed curve, and for large $p$ where the Julia set is completely disconnected. In both cases the Hausdorff dimension is calculated in perturbation theory and numerically. An expression for the rate at which points escape from the neighborhood of the Julia set is derived and tested in a numerical simulation of the escape.
\end{abstract}

KEY WORDS: Chaos; dynamical systems; fractal dimension; escape rate; Julia set.

\section{INTRODUCTION}

\subsection{Definition of Julia Set}

In dynamical systems theory ${ }^{(1)}$ one studies the effect of a deterministic process which takes a point $\mathbf{r}$ into a new point $\mathbf{r}^{\prime}=f(\mathbf{r})$. One is particularly interested in classifying points into sets which remain invariant under the mapping $f$. Iterating $f$ corresponds to studying the long-time behavior of a dynamical system. All points of an invariant set show similar long-time behavior. Some sets show extremely complicated long-time behavior and for this reason are called "strange."(2)

Strange sets which appear in nature have proven to be difficult to study quantitatively. For this reason we focus our attention here on a

\footnotetext{
1 The University of Chicago, The James Franck Institute, Chicago, Illinois 60637.
} 
problem which shares many features of more realistic models, yet is relatively tractable. The simplification is that we study a mapping of the plane which can be represented as an analytic mapping of a single complex variable. We shall treat the case in which $f$ is a polynomial

$$
f(z)=z^{Q}+p
$$

with special emphasis on the case $Q=2$.

Consider first the simple mapping

$$
f(z)=z^{Q}
$$

We find three invariant sets associated with the mapping (1.2). Initial points inside the unit circle are attracted to a stable fixed point at 0 . Initial points outside the unit circle are attracted to a stable fixed point at infinity. The unit circle itself is the third invariant set.

The Julia set of a mapping is defined as the closure of the set of unstable cycles. ${ }^{(3-5)}$ The mapping (1.2) has stable fixed points at 0 and $\infty$. Any unstable cycle must therefore lie on the unit circle. Indeed, the points

$$
\begin{gathered}
z\left(t_{j}\right)=e^{2 \pi i i_{j}} \\
t_{j}=\frac{j}{Q^{n}-1} \\
j=0,1,2, \ldots, Q^{n}-2
\end{gathered}
$$

are the elements of all unstable cycles of length $n$. Note that these points are dense on the unit circle and hence the unit circle is the Julia set of the mapping (1.2). The notation Fix $f^{n}$ will be used to denote the set of unstable fixed points of $f^{n}$.

One can also describe the Julia set in terms of the inverses $f_{\tau_{1} \tau_{2} \ldots \tau_{n}}^{(-n)}$ of $f^{n}$. The $Q^{n}$ inverse functions are labeled by the values of $\tau_{1}, \tau_{2}, \ldots, \tau_{n}$ and are defined recursively by

$$
f_{\tau_{0} \tau_{1} \ldots \tau_{n}}^{-(n+1)}=f_{\tau_{0}}^{-1} \circ f_{\tau_{1} \tau_{2} \ldots \tau_{n}}^{-n}
$$

where the symbol $\tau_{i}$ defines a particular branch of the inverse. When $f$ is a quadratic function the inverses are

$$
\begin{aligned}
f_{\tau}^{-1}(z) & =\tau(z-p)^{1 / 2} \\
f_{\tau_{1} \tau_{2} \ldots \tau_{n}}^{-n}(z) & =\tau_{1}\left\{-p+\tau_{2}\left[-p+\cdots+\tau_{n}(z-p)^{1 / 2}\right]^{1 / 2}\right\}^{1 / 2}
\end{aligned}
$$

where $\tau_{i}= \pm 1$.

If $z$ is a member of the Julia set of $f$, then the inverses $f^{-n}(z)$ are also members. In fact as $n \rightarrow \infty$ the set $f^{-n}(z)$ approaches the entire Julia set with the same distribution ${ }^{(3,12)}$ as the set Fix $f^{n}$. 

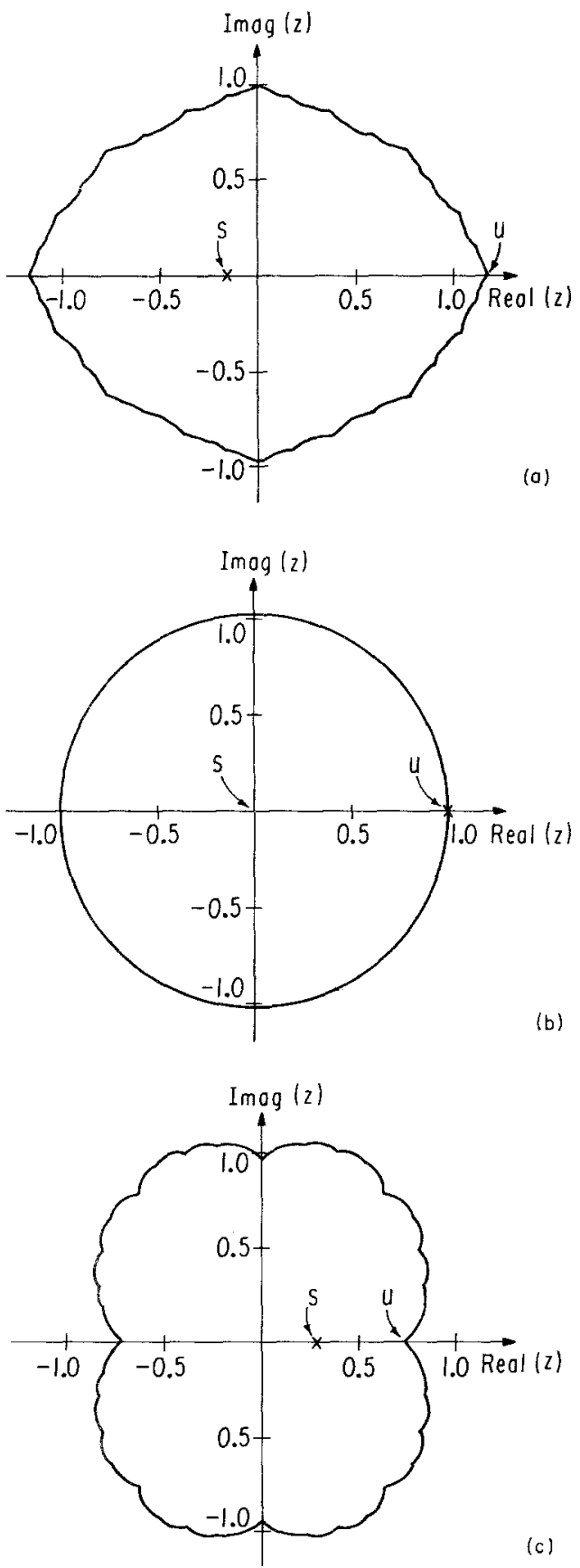

Fig. 1. Julia sets of the mapping $f(z)=z^{2}+p$ for small $p$. (a) $p=-0.2$, (b) $p=0$, (c) $p=0.2$. In case (b) the Julia set is the unit circle. Stable and unstable fixed points are denoted $s$ and $u$, respectively. 


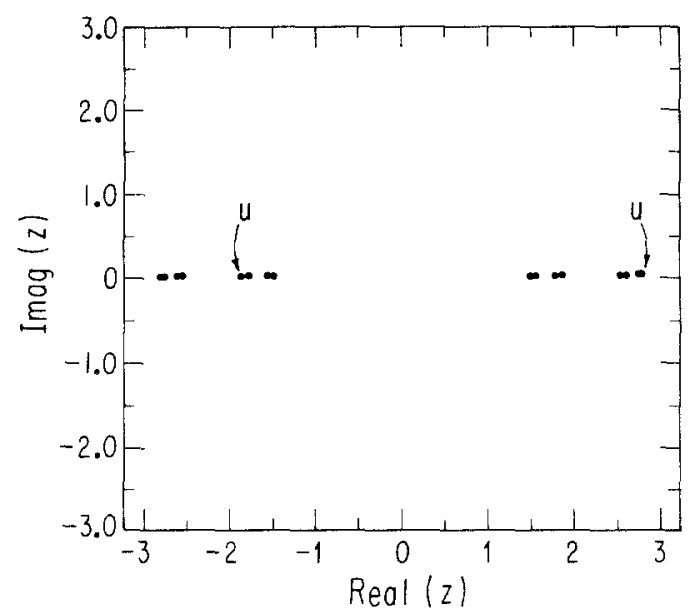

Fig. 2. Julia set of the mapping $f(z)=z^{2}-5$. The unstable fixed points are denoted $u$.

\subsection{Further Examples of Julia Sets}

The unit circle is an unusually simple example of a Julia set. More complicated sets are found when the parameter $p$ in (1.1) is taken to be nonzero. We will study the cases of small and large $p$ in detail. When $p$ is small the Julia set remains topologically a circle. That is, it can still be parametrized by a continuous function $z(t)$ where $t \in[0,1)$ and $z(0)=z(1)$. Points outside the Julia set still flow to infinity while points inside the Julia set flow to the stable fixed point $z^{*} \approx p$. Figure 1 shows examples of such Julia sets. Notice that the curves have bumps on top of bumps on top of bumps. Our major goal in this paper is to characterize the geometry of this curve. Sullivan $^{(6)}$ has proven that these sets have Hausdorff dimensions greater than $\mathbf{1}$.

As $|p|$ increases the topology of the Julia set undergoes many changes. ${ }^{(7,8)}$ For large $|p|$ the topology simplifies. All cycles are unstable except the point at infinity which attracts the entire complex plane except the Julia set. The Julia set is completely disconnected, as can be seen in Fig. 2. Points of the Julia set cluster around the unstable fixed points at $z^{*} \approx(-p)^{1 / Q}$.

\subsection{Measurable Quantities}

We seek ways to characterize Julia sets. One commonly used characterization is the Hausdorff dimension $D_{H}$, which describes geometrical properties of the set. An attribute of Julia sets which describes dynamics in addition to geometry is the escape rate. To define it, cover the Julia set with 
a region which does not contain any stable cycle. Spray a large number of points onto this area and iterate them. The number of points which remain in the area after $n$ iterations decreases exponentially for large $n$. The rate of decrease is independent of the area which we choose and is called the escape rate of the Julia set.

In Section 2 of this paper we show how perturbation calculations for small and large $p$ can be carried out. In Section 3 we specialize to the subject of escape rate, derive formulas with which the escape rate can be calculated, and test these formulas by direct numerical experiments. Finally, in Section 4 we utilize a theorem of Ruelle ${ }^{(9)}$ to compute the Hausdorff dimension numerically, and in perturbation theory.

\section{PERTURBATION THEORY}

When $p=0$ the Julia set consists of the unit circle. Parametrizing this curve as $z(t)=e^{2 \pi i t}$ induces the linear map $t \rightarrow Q t$ on $t$

$$
f(z(t))=z(Q t)
$$

When $p$ is nonzero but small enough so that the Julia set is topologically equivalent to a circle we can still parametrize the curve so that $(2.1)$ is satisfied. ${ }^{(3,5)}$ The function $z(t)$ can be formally expanded in powers of $p$

$$
z(t)=e^{2 \pi i t}\left[1+p U_{1}(t)+p^{2} U_{2}(t)+\cdots\right]
$$

where each $U_{m}(t)$ is periodic with period 1. Substituting (2.2) into (2.1) and equating terms with the same order in $p$ yields, up to second order, the following equations:

$$
\begin{aligned}
& U_{1}(Q t)-Q U_{1}(t)=e^{-2 \pi i Q t} \\
& U_{2}(Q t)-Q U_{2}(t)=\frac{Q(Q-1)}{2} U_{1}^{2}(t)
\end{aligned}
$$

Notice that the basic linear equation

$$
\phi(Q t)-Q \phi(t)=e^{-2 \pi i t}
$$

has the solution

$$
\phi(t)=\frac{-1}{Q} \sum_{l=0}^{\infty} Q^{-l} e^{-2 \pi i Q^{\prime} t}
$$

Therefore,

$$
\begin{aligned}
& U_{1}(t)=\phi(Q t) \\
& U_{2}(t)=\frac{Q(Q-1)}{2} \sum_{l_{1}, l_{2}=1}^{\infty} Q^{-\left(l_{1}+l_{2}\right)} \phi\left(\left(Q^{l_{1}}+Q^{l_{2}}\right) t\right)
\end{aligned}
$$


In general, the $m$ th-order terms obey an equation of the form

$$
U_{m}(Q t)-Q U_{m}(t)=H_{m}\left(t, U_{1}(t), U_{2}(t), \ldots, U_{m-1}(t)\right)
$$

so that one can solve recursively for all higher-order terms.

This knowledge of $z(t)$ allows us to do formal perturbative calculations of various properties of the Julia set. For example, one quantity that will prove useful for understanding both the fractal dimension and the escape rate is

$$
A_{n}(D)=\sum_{z \in \text { Fix } f^{n}}\left|\frac{d f^{n}}{d z}\right|^{-D}
$$

In what follows we extend to third order in $p$ a calculation first carried out by Ruelle ${ }^{(9)}$ to second order. Relations (1.3) and (2.1) allow us to express the set Fix $f^{n}$ as

$$
\text { Fix } f^{n}=\left\{z\left(t_{j}\right): t_{j}=\frac{j}{Q^{n}-1}, j=0,1, \ldots, Q^{n}-2\right\}
$$

Using the fact that

$$
\left.\frac{d f^{n}}{d z}\right|_{z=z\left(t_{j}\right)}=\prod_{m=0}^{n-1} f^{\prime}\left(z\left(Q^{m_{t}}\right)\right)=Q^{n}\left[\prod_{m=0}^{n-1} z\left(Q^{m_{t_{j}}}\right)\right]^{Q-1}
$$

and introducing the notation

$$
\langle G(t)\rangle_{n}=\frac{1}{Q^{n}-1} \sum_{j=0}^{Q^{n}-2} G\left(t_{j}\right)
$$

we can write $A_{n}(D)$ as

$$
A_{n}(D)=Q^{-n D}\left(Q^{n}-1\right)\left\langle\prod_{m=0}^{n-1} \mid z\left(Q^{m_{t}}\right)^{-(Q-1) D}\right\rangle_{n}
$$

By substituting (2.2) into (2.14), $A_{n}(D)$ can be formally expanded in powers of $p$. The evaluation of the averages can then be performed through the use of the identity

$$
\left\langle e^{2 \pi i m t}\right\rangle_{n}=\left\{\begin{array}{lll}
1, & m \equiv 0 & \bmod Q^{n}-1 \\
0, & m \neq 0 & \bmod Q^{n}-1
\end{array}\right.
$$

We have carried out this calculation to third order in $p$, and find that for all $n \geqslant 4$

$$
\begin{aligned}
A_{n}(D)=Q^{-n D}\left(Q^{n}-1\right) & \left\{1+p \bar{p} \frac{D^{2} n}{4}+\delta_{Q, 2}\left(p^{2} \bar{p}+\bar{p}^{2} p\right)\right. \\
& \left.\times\left[\left(\frac{D^{2}}{4}\right) \frac{n}{2^{n}-1}+\left(\frac{D^{3}}{16}+\frac{D^{2}}{8}\right) n\right]+O\left(p^{4} n^{2}\right)\right\}
\end{aligned}
$$


The structure of Eq. (2.16) is determined by the invariance of geometrical properties of the Julia set under certain coordinate changes. First, consider the reflection

$$
h(z)=\bar{z}
$$

Note that

$$
h \circ f \circ h^{-1}(z)=z^{Q}+\bar{p}
$$

Since $A_{n}(D)$ must be invariant under reflection, the perturbation expansion must be symmetric under interchange of $p$ and $\bar{p}$. Next, Eq. (2.15) prohibits the appearance of terms proportional to $\left(p^{r}+\bar{p}^{r}\right)$ for sufficiently large $n$. Finally, consider the family of rotations

$$
h_{r}(z)=e^{2 \pi i r /(Q-1)} z, \quad r=0,1, \ldots, Q-2
$$

Note that

$$
h_{r} \circ f \circ h_{r}^{-1}(z)=z^{Q}+e^{2 \pi i r /(Q-1)} p
$$

Since $A_{n}(D)$ must be invariant under rotations, the perturbation expansion must be symmetric under rotations of $p$ by $h_{r}$. Thus terms of the form $\left(p^{\prime} \bar{p}^{s}+\bar{p}^{\prime} p^{s}\right)$ are allowed only if $(r-s) /(Q-1)$ is an integer.

For large $p$ the Julia set is clustered around the $Q$ unstable fixed points at $z \approx(-p)^{1 / Q}$. If we consider only the leading behavior then

$$
A_{n}(D) \sim Q^{n(1-D)}|p|^{-n D / Q}
$$

\section{ESCAPE RATE}

The escape rate is defined by the following algorithm: spray a large number of points, $N_{0}$, in some neighborhood of the Julia set. Iterate them $n$ times and count the number of points remaining in $\Gamma, N_{n}$. As $n$ gets large, $N_{n}$ decays exponentially:

$$
N_{n} \sim q^{-n}
$$

where $q$ is the escape rate.

To derive an expression for $q$, let us assume a uniform distribution of points $\rho(x, y)$ which is non-zero in $\Gamma$ and zero otherwise,

$$
\int_{\Gamma} \rho(x, y) d x d y=1
$$

the number of points remaining in $\Gamma$ after $n$ iterations is

$$
B_{n}=\int_{\Gamma} d^{2} \mathbf{r}^{\prime} d^{2} \mathbf{r} \rho(\mathbf{r}) \rho\left(\mathbf{r}^{\prime}\right) \delta\left[\mathbf{r}^{\prime}-f^{n}(\mathbf{r})\right]=\int_{\Gamma} d^{2} \mathbf{r} \rho(\mathbf{r}) \rho\left[f^{n}(\mathbf{r})\right]
$$

with $\mathbf{r} \equiv(x, y)$. Since $\rho\left[f^{n}(\mathbf{r})\right] \neq 0$ implies $\rho(\mathbf{r}) \neq 0$, but not vice versa, it will 
be more useful to look at the inverses $f_{\tau_{1} \ldots \tau_{n}}^{-n}(\mathbf{r})$ of $f^{n}(\mathbf{r})$. Since $f$ is an analytic function, the Jacobian of the transformation from $\mathbf{r}$ to $\mathbf{r}^{\prime}$ is

$$
J=\left|\frac{d f^{(n)}(z)}{d z}\right|_{z=f_{\tau_{1} \ldots}^{-n} \tau_{n}\left(z^{\prime}\right)}^{2}
$$

with $z=x+i y$. Therefore

$$
B_{n}=\sum_{\left\{\tau_{i}\right\}} \int_{\Gamma} d^{2} \mathbf{r}^{\prime} \frac{\rho\left(\mathbf{r}^{\prime}\right) \rho\left[f_{\tau_{1} \ldots \tau_{n}}^{-n}\left(\mathbf{r}^{\prime}\right)\right]}{\left|d f^{n} / d z\right|_{z=f_{\tau_{1} \ldots \tau_{n}}^{-n}\left(z^{\prime}\right)}^{2}}
$$

Note that for all $\mathbf{r}^{\prime}$ in $\Gamma$ and in the limit $n \rightarrow \infty$, the value of $z=$ $f_{\tau_{1} \ldots \tau_{n}}^{-n}\left(z^{\prime}\right)$ is independent of $z^{\prime}$. (or $\mathbf{r}^{\prime}$ ), and is distributed according to the density of unstable cycles. ${ }^{(3)}$ Thus

$$
B_{n} \simeq A_{n}(2)=\sum_{z \in \text { Fix } f^{n}}\left|\frac{d f^{n}}{d z}\right|^{-2}
$$

When $p<-2$ the Julia set lies on the real axis and we can define a one-dimensional escape rate through

$$
B_{n} \simeq A_{n}(1)=\sum_{z \in \mathrm{Fix} f^{n}}\left|\frac{d f^{n}}{d z}\right|^{-1}
$$

The escape rate is then

$$
q=\lim _{n \rightarrow \infty} A_{n}^{-1 / n}(D)
$$

We may use Eq. (2.16) for $A_{n}(D)$ to express $q$ as a function of $p$ (for small $p$ ):

$$
q=Q\left\{1-p \bar{p}-\delta_{Q, 2}\left(p^{2} \bar{p}+\bar{p}^{2} p\right)\right\}
$$

For $p \rightarrow-\infty$ and $Q=2$, we may use the fact that the Julia set is clustered around $x= \pm(|p|)^{1 / 2}$ to derive

$$
q= \begin{cases}(|p|)^{1 / 2}, & D=1 \\ 2|p|, & D=2\end{cases}
$$

We have tested Eq. (3.8) in numerical experiments done on the mapping $f(z)=z^{2}+p$ at $p \approx 0$ and $p \lesssim-2$ (for $D=1$ and $D=2$ ). The simulations were done by randomly distributing $N_{0}$ points $\left(N_{0}=10^{5}, 10^{6}\right)$ in some neighborhood $\mathrm{T}$ of the Julia set and counting the "survivors" after $n$ iterations. Following a transient period of $\sim 10$ iterations, an exponential decay is observed to which a decay rate $q$ is best-fitted. In the onedimensional case it is possible to measure the escape rate by a more accurate procedure. In that case, the neighborhood $\Gamma$ of the Julia set is a segment on the real axis. Consider its back-iterates. Iterating by $f^{-1}$ 
Table I. Theoretical and Experimental Escape Rates ${ }^{a}$

\begin{tabular}{clcc}
\hline & $p$ & $q$-theoretical & $q$-experimental \\
\hline \multirow{4}{*}{$2-D$} & 0.1 & 1.9741 & 1.971 \\
& 0.15 & 1.9282 & 1.930 \\
& 0.2 & 1.8139 & 1.813 \\
& -2.015025 & 2.125 & 2.129 \\
& -2.075625 & 2.339 & 2.341 \\
& -2.1525 & 2.545 & 2.548 \\
& -2.230625 & 2.732 & 2.717 \\
& & & \\
& -2.4725 & 1.3020 & 1.3022 \\
$1-D$ & -3.75 & 1.7384 & 1.7380 \\
& -20 & 4.3888 & 4.3887 \\
& -48.75 & 6.9278 & 6.9285 \\
& -90 & 9.4458 & 9.4473 \\
\hline
\end{tabular}

${ }^{a}$ Theoretical rates were obtained through Eq. (3.8). $D=2$ experimental rates were obtained through iterating an ensemble of points. $D=1$ experimental rates were obtained through Eq. (3.11).

generates two segments which by $f$ map into $\Gamma$. We denote by $\Gamma_{1}$ the total measure (length) of these segments. Iterating by $f^{-2}$ generates four segments whose total length we denote by $\Gamma_{2}$, etc. ${ }^{(10)}$ Clearly the escape rate is

$$
q=\lim _{n \rightarrow \infty} \frac{\Gamma_{n-1}}{\Gamma_{n}}
$$

Formulas (3.8) and (3.11) were evaluated at $n=11$. Our experimental results are summarized in Table I. The good agreement between the theoretical prediction Eq. (3.8), and the numerical results thus validates our formulation of the escape rate.

\section{HAUSDORFF DIMENSION}

A theorem of Ruelle ${ }^{(9)}$ provides a useful formula for computing Hausdorff dimensions. Ruelle proved that when $D$ equals the Hausdorff dimension of the Julia set of $f$ the series

$$
\zeta(u)=\exp \sum_{n=1}^{\infty} A_{n}(D) \frac{u^{n}}{n}
$$

extends to a meromorphic function with a simple pole at $u=1$ and no other poles or zeros inside the unit disk. Taking the logarithm of Eq. (4.1), we see that a necessary condition for $D_{H}$ to be the Hausdorff dimension is

$$
\lim _{n \rightarrow \infty} A_{n}\left(D_{H}\right)=1
$$


Table II. Comparison of Numerical Calculations of $D_{H}{ }^{a}$

\begin{tabular}{rcc}
\hline$n$ & $A_{n}\left(D_{n}\right)=1$ & $A_{n}\left(D_{n}\right)=1-2^{-n}$ \\
\hline 5 & 0.991808 & 1.00096909 \\
6 & 0.997190 & 1.00097653 \\
7 & 0.999363 & 1.00097966 \\
8 & 1.000275 & 1.00098091 \\
9 & 1.000668 & 1.00098140 \\
10 & 1.000841 & 1.00098160 \\
11 & 1.000918 & 1.00098168 \\
12 & 1.000952 & 1.00098171 \\
\hline
\end{tabular}

${ }^{a}$ Calculations carried out for $p=0.05$.

Inspecting Eq. (2.16) we see that (4.2) is satisfied if

$$
D_{H}=1+\frac{p \bar{p}}{4 \ln Q}+\delta_{Q, 2} \frac{3\left(p^{2} \bar{p}+\bar{p}^{2} p\right)}{16 \ln Q}
$$

The first two terms in this formal power series were obtained by Ruelle.

We have checked Eq. (4.3) numerically. Table II shows typical results of a calculation for mapping $f(z)=z^{2}+p, p=0.05$. A straightforward calculation of $D_{H}$ is shown in the second column. Here we define $D_{n}$ by $A_{n}\left(D_{n}\right)=1$. Note that $D_{n}-1$ has converged to just one significant figure when $n=12$.

We can speed the convergence of $D_{n}$ to $D_{H}$ by employing the results of perturbation theory. Inserting the result (4.3) into our expression for $A_{n}(D)$ (2.16) we find

$$
A_{n}\left(D_{H}\right)=1-Q^{-n}+O\left(p^{3} Q^{-n}\right)
$$

This rather slow convergence of $A_{n}\left(D_{H}\right)$ to 1 is the cause of the slow convergence of $D_{n}$ to $D_{H}$. To speed up this convergence, we define $D_{n}$ by

$$
A_{n}\left(D_{n}\right)=1-Q^{-n}
$$

The last column in Table II is obtained in this manner.

With this numerical technique we can check the correctness of Eq. (4.3). We assume, on the basis of symmetries discussed in Section 2,

$$
D_{H}(p)=1+a_{2}|p|^{2}+a_{3}|p|^{2} \operatorname{Re}(p)+\cdots
$$

This form is consistent with numerical studies by L. Garnett (unpublished). We can determine $a_{2}$ and $a_{3}$ numerically by

$$
a_{2}=\lim _{p \rightarrow 0^{+}} \frac{D_{H}(p)+D_{H}(-p)-2}{2 p^{2}}
$$


and

$$
a_{3}=\lim _{p \rightarrow 0^{+}} \frac{D_{H}(p)-D_{H}(-p)}{2 p^{3}}
$$

Results of this calculation are shown in Table III.

The Hausdorff dimension can be computed in the limit of large $p$. Setting $A_{n}\left(D_{H}\right)=1$ in Eq. (2.21) yields

$$
D_{H}=\frac{Q \ln Q}{\ln |p|}
$$

It is interesting to note that in the limit as $p \rightarrow-\infty$ we can compute $D_{H}$ without using Ruelle's formula. Observe that the Julia set is contained in the interval

$$
c_{1}=\left[\frac{1}{2}-\left(\frac{1}{4}-p\right)^{1 / 2}, \frac{1}{2}+\left(\frac{1}{4}-p\right)^{1 / 2}\right]
$$

Taking the preimages of this interval of $f$ we note that the Julia set is contained in

$$
c_{2}=c_{1}-\left(\left[\frac{1}{2}-p-\left(\frac{1}{4}-p\right)^{1 / 2}\right]^{1 / 2},\left[\frac{1}{2}-p+\left(\frac{1}{4}-p\right)^{1 / 2}\right]^{1 / 2}\right)
$$

In general we find that the Julia set is contained in $C_{n}$ where $C_{n}$ consists of $N(\lambda)=2^{n}$ intervals with maximum length $\lambda \sim|p|^{-n / 2}$. Employing Mandelbrot's $^{(11)}$ formula

$$
\lambda \cdot N(\lambda) \sim \lambda^{1-D}
$$

yields Eq. (4.9) with $Q=2$.

A final observation is that the escape time and Hausdorff dimension are independent characterizations of a Julia set. To see this, assume they

Table III. Test of Perturbation Theory for $D_{H}$ (4.3)

\begin{tabular}{ccc}
\hline$p$ & $\left(D^{+}+D^{-}-2\right) / 2|p|^{2}$ & $\left(D^{+}-D^{-}\right) / 2|p|^{3}$ \\
\hline \pm 0.1 & 0.379422 & 0.593 \\
\pm 0.01 & 0.360845 & 0.542 \\
\pm 0.001 & 0.360677 & 0.540 \\
theoretical & 0.360674 & 0.541 \\
\hline
\end{tabular}

${ }^{a} D^{+}$and $D^{-}$are determined by $A_{12}\left(D^{+}\right)=A_{12}\left(D_{H}\right)$ for the map $f(z)=z^{2}+p$ and $A_{12}\left(D^{-}\right)=A_{12}\left(D_{H}\right)$ for the map $f(z)=z^{2}-p$. The linear combinations of $D^{+}$and $D^{-}$in the second and third columns pick out the coefficients of $|p|^{2}$ and $|p|^{2} \operatorname{Re}(p)$, respectively. The bottom row gives the predicted values from Eq. (4.3). 
are related by

$$
q=g\left(D_{H}-1\right)
$$

for some function $g$. Expanding (4.13) in powers of $p$ we notice that for (4.13) to hold we must have the ratio of quadratic coefficients in $q$ and $D_{H}$ equal to the ratio of cubic coefficients. Inspecting equations (3.9) and (4.3) we see that this is not the case.

\section{ACKNOWLEDGMENT}

We wish to thank D. Ruelle and D. Sullivan for useful correspondence and discussions. This work was supported in part by NSF Grant No. 80-20609. S. J. S. and M. W. wish to acknowledge the support, respectively, of an R. R. McCormick Fellowship and a W. R. Harper Fellowship.

\section{REFERENCES}

1. P. Collet and J.-P. Eckmann, Iterated Maps of the Interval as Dynamical Systems (Birkhauser, Boston, 1980).

2. E. Ott, Rev. Mod. Phys. 53:655 (1981).

3. For a review see H. Brolin, Ark. Mat. 6:103 (1965).

4. G. Julia, J. Math. Pures Appl. 8:47 (1918).

5. P. Fatou, Bull. Soc. Math. France 47:161 (1919); 48:33 (1920).

6. D. Sullivan, Proceedings of the International Conference on Dynamical Systems, Rio de Janeiro (1981).

7. B. Mandelbrodt, Ann. N. Y. Acad. Sci. 357:249 (1980).

8. A. Douady and J. H. Hubbard, C. R. Acad. Sci. Paris 294:123 (1982).

9. D. Ruelle, submitted to J. Ergodic Theory Dyn. Sys. (1982).

10. E. Domany, S. Alexander, D. Bensimon, and L. P. Kadanoff, to appear in Phys. Rev. B.

11. B. Mandelbrodt, The Fractal Geometry of Nature (Freeman, San Francisco, 1982).

12. M. Barnsley, private communication (1983). 